\title{
THE NATURE OF DIABETIC ACIDOSIS
}

\author{
By JOHN P. PETERS, DAVID M. KYDD, ANNA J. EISENMAN AND \\ PAULINE M. HALD
}

(From the Department of Internal Medicine of Yale University and the Medical Service of the New Haven Hospital, New Haven)

(Received for publication November 25, 1932)

Since the discovery that ketone acids are produced in the body and accumulate in the blood to excess in severe diabetes, general opinion has held that the accumulation of these chemical compounds is responsible for the syndrome known as diabetic coma. The general application of the term acidosis to the condition is in itself sufficient evidence of the importance which is attached to this disorder of metabolism. Difference of opinion seems to have been restricted chiefly to the question of the relative parts played by the ketone bodies as such and by the reduction of blood alkali and diminution of $\mathrm{pH}$ which they caused. In favor of direct action of the ketones have been cited the demonstrated anesthetic effects of acetone and diacetic acid, while the adherents of the acidosis theory depend upon experiments in which various workers, beginning with Walter (1) have demonstrated the disastrous, even lethal, effects of injections of massive doses of mineral acids.

With the appearance and application of accurate and practical methods for the determination of blood bicarbonate and $\mathrm{pH}$ it has become increasingly apparent that alkali deficits of the magnitude found in diabetic acidosis, when they are produced experimentally or occur in the course of other diseases, are not necessarliy attended by a syndrome resembling that of diabetic acidosis. This has, perhaps, given more weight to the arguments of those who would hold that acetone and diacetic acid, by their anesthetic and poisonous effects, are responsible for the symptoms and fatalities. On the other hand there is but the scantiest positive quantitative evidence to support such a theory. The anesthetic actions of acetone and diacetic acid are notoriously slight, but hard to ascertain with certainty because of the ease with which normal animals excrete or oxidize these compounds. Ketosis unassociated with the other metabolic disorders of diabetes never attains so great an intensity. The most convenient experimental animals when rendered diabetic by pancreatectomy or phlorizin do not develop ketosis comparable in severity to that seen in humans with diabetic coma. Chemical analyses have demonstrated no exact correlation between the concentration of ketones in the blood and the profundity of coma in diabetic patients (20). 
Even since it has been apparent that alkali deficit is not the chief cause of symptoms, it has been held responsible for the persistence of certain metabolic abnormalities by the adherents of alkaline therapy. Retarded carbohydrate utilization (2) and delayed recovery of consciousness (3) have both been attributed to the effects of serum bicarbonate deficiency.

Until the advent of insulin it was impossible to dissociate the various metabolic disturbances of diabetic acidosis in order to analyze their individual roles in the production of symptoms. Insulin has enabled the clinician to reestablish carbohydrate combustion and consequently ketone oxidation with great rapidity and thus to test the hypotheses which have been discussed above. So firmly, however, have earlier preconceptions become established, that variables other than the conventional ones, sugar, serum bicarbonate and ketosis, have been given little consideration.

It has already been pointed out in other articles that carbohydrate combustion, under the influence of insulin, may be accelerated to such an extent that the blood sugar falls to normal or hypoglycemic levels while serum bicarbonate still remains within the low range in which coma may be expected; in one case as low as 7.3 m.eq. The idea that alkali deficit retards or inhibits glucose oxidation is hardly compatible with these facts, even if physiologic support for such a theory were not entirely lacking (4). Haldane (5) and others $(6,7,8)$ have, indeed, adduced evidence that acidifying measures stimulate and alkalinizing measures retard carbohydrate combustion. That alkalinizing measures may cause ketosis has been repeatedly demonstrated $(5,6,9,10,11)$. It has further been shown that the state of consciousness is but roughly related to the serum bicarbonate. Consciousness may be retained or recovered while bicarbonate is extremely low; while stupor or coma may develop or persist when it is only moderately reduced. Finally the importance and significance of general dehydration and hemoconcentration in diabetic acidosis have been pointed out.

In the present paper the nature and causes of bicarbonate deficits and other electrolyte disturbances in the serum are analyzed. Their clinical significance and therapeutic implications will be discussed in subsequent communications.

\section{METHODS AND MATERIAL}

The clinical material consists of $\mathbf{4 0}$ patients who were admitted to the hospital one or more times with severe diabetic acidosis. Studies of blood sugar, serum bicarbonate and usually total serum electrolyte equilibria, were made at the time of admission and at intervals in the course of recovery-or, in a few fatal cases, before death-by the methods usually employed in this laboratory (12). 


\section{RESULTS}

There is little correlation between blood sugar and bicarbonate at the time of admission, when acidosis is most extreme. To be sure, there is a tendency for very high blood sugars to coincide with low serum bicarbonate. Initial blood sugars of $500 \mathrm{mgm}$. per cent or more occurred only when bicarbonate was below 11 m.eq. On the other hand the most extreme reductions of bicarbonate were associated with blood sugars between 300 and $450 \mathrm{mgm}$. per cent. Exact comparison of ketosis and serum bicarbonate is impossible because blood was not analyzed for ketone bodies and urine was subjected only to the qualitative nitroprusside test. Certainly the intensity of ketosis as measured by this rough method bears no relation to the clinical severity of the acidosis nor to the degree of bicarbonate reduction. In fact in the most extreme cases of acidosis the initial nitroprusside reaction was of ten quite moderate and frequently became more intense as the condition improved and serum bicarbonate rose under treatment. The explanation for this paradox is probably to be found in the low concentrating powers of the kidney at the height of acidosis, to which attention has already been called (13). The same patients frequently had initially high blood nonprotein nitrogen, which fell rapidly as diuresis was established. What is at first sight more surprising is the fact that ketonuria often disappeared completely while there was still a considerable alkali deficit. In 13 cases observations of bicarbonate were carried out until ketonuria had disappeared or become negligible and urine specimens were collected and analyzed at sufficiently frequent intervals to determine the time of its disappearance within reasonable limits. The serum bicarbonate in these cases, when ketonuria had been eliminated, ranged from 9.7 to $23.1 \mathrm{~m}$.eq. In every instance, moreover, because the urine represented the accumulation of a considerable period, the actual time of disappearance of ketonuria must have coincided with a still lower bicarbonate level. This can be illustrated by certain extreme examples. Case 71956 was admitted to the hospital at 9:20 P. M. with a serum bicarbonate of $3.3 \mathrm{~m} . e q$. and heavy acetone. The serum bicarbonate at 3:00 A. M. was 6.4 and at 7:30 A. M. 9.7 m.eq. It is, therefore, conservative to say that ketonuria cleared with bicarbonate at 9.7 ; it may have disappeared when bicarbonate was only 6.4. Case A-1126 was admitted at 4:00 P. M. with a serum bicarbonate of 3.3 m.eq. and heavy acetonuria. Urine could not be collected again until 8:00 A. M. next morning, when it gave no nitroprusside reaction. At this time the bicarbonate was 11.6 m.eq. Another case has been cited in the previous paper (14). Such a dissociation between ketonuria and alkali deficit would be impossible if the latter were due simply to displacement of bicarbonate by ketone acids, as is too generally implied in discussions of the subject.

The nature of the alkali deficit can be tested in another manner. In 
a considerable number of cases the total acid base equilibrium of the serum was determined. Theoretically the total base concentration, expressed in combining equivalents, should equal the sum of the concentrations of the serum acids, protein, phosphate, chloride, bicarbonate, sulfate and organic acids. If total base, protein, phosphate, chloride, and bicarbonate are determined, the concentration of organic acid can be calculated by the equation

$$
\begin{array}{r}
{[\mathrm{B}]-\left([\text { protein }]+\left[\mathrm{HPO}_{4}\right]+\left[\mathrm{H}_{2} \mathrm{PO}_{4}\right]+[\mathrm{Cl}]+\underset{ }{[}=\left[\mathrm{HCO}_{3}\right]\right)} \\
=\left[\mathrm{SO}_{4}\right]+\text { organic acids. }
\end{array}
$$

As there is no reason for believing that serum sulfate rises in diabetic acidosis, the undetermined acid fraction must vary with the concentration of organic acids. In diabetic acidosis this organic acid fraction is greatly augmented by the accession of ketone acids and major variations of the undetermined acid concentration are probably due to fluctuations of ketosis. If bicarbonate reduction were due chiefly to ketosis, then, there should be a very clear inverse or reciprocal relation between bicarbonate and undetermined acids. Figure 1, however, shows that in the

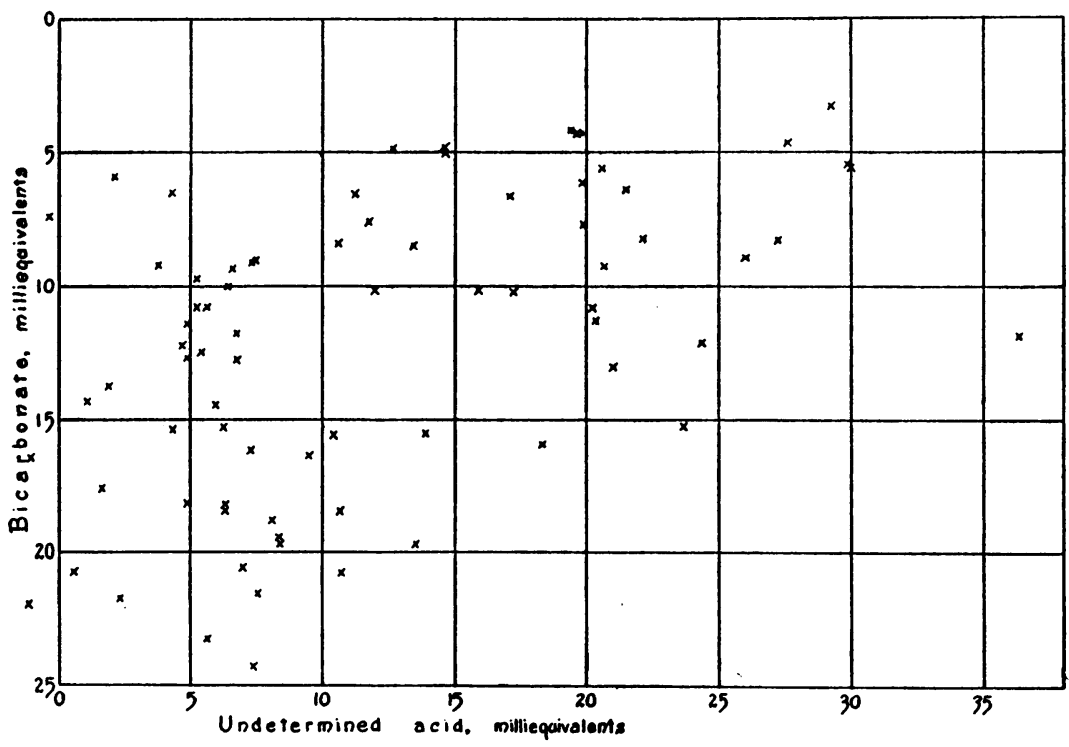

Fig. 1. The Relation of the Concentration of Bicarbonate to That of Undetermined Acids (Presumably Chiefly Ketones) in Serum in Diabetic Acidosis.

data under discussion the correlation between the two variables, though demonstrable, is extremely rough.

The reduction of bicarbonate can, then, be only partly due to displacement by ketone acids. In part it is due also to actual deficiency of 
base in the serum, brought about by the excretion of sodium and other fixed bases to neutralize those ketone acids for which ammonia is not provided. The reasons for the base deficit and the sequence of events which lead to it are probably quite similar to those described by Gamble, Ross and Tisdall (15) in starvation and have been discussed in relation to diabetic acidosis elsewhere (16). It must be obvious from the equation above that the concentration of total base in the serum sets a limit to the concentration of acids and especially of the weak, volatile acid, bicarbonate. It has, furthermore, been demonstrated that, in the majority of instances, chloride as well as bicarbonate is reduced in diabetes. It looks as if both chloride and bicarbonate yielded base for the neutralization of ketone acids. Reduction of bicarbonate, then, is due not only to displacement of this radicle from the base with which it was formerly combined, but also to a deficiency of base itself. On the other hand, alternative displacement of chloride may mitigate the contraction of bicarbonate to a greater or less extent. Dehydration also diminishes the apparent bicarbonate deficiency. If the volume of fluid in which bicarbonate is dissolved contracts, while the actual quantity of bicarbonate remains constant, the concentration of bicarbonate in the remaining fluid must increase.

In Figure 2 an attempt has been made to depict in a diagrammatic manner the principal changes of electrolytes in the body fluids in severe diabetic acidosis. For purposes of simplification alterations of protein and phosphate concentrations have been neglected; only bicarbonate, chloride and the undetermined acid fraction (the latter serving as a measure of ketosis) have been considered. The vertical height of each column represents concentration of acids, the width of the column indicates the volume of the body fluids. As the sums of the basic and acid equivalents must always be equal, the total height of each column represents the concentration of base.

The left hand column illustrates conditions in the average normal subject, ${ }^{1}$ where the concentration of chloride is 102.6 m.eq., of bicarbonate 26.4 m.eq., of undetermined acids $3.6 \mathrm{~m}$.eq. and of base $152.1 \mathrm{~m}$.eq. The right hand column, except that the loss of water has been arbitrarily taken as $\mathbf{2 5}$ per cent of the volume of the body fluids, gives a fair picture of the electrolyte changes in the average patient with diabetic acidosis. By taking the average initial values for each electrolyte component of the serum in the series of cases under consideration, the average electrolyte picture of diabetic acidosis has been derived. The average of $\mathbf{4 2}$ observations of bicarbonate was 8.9 m.eq.; the average of 41 chloride determinations 95.0 m.eq.; the average of 29 total bases was 142.9 m.eq.;

1 The values are derived from a large series of analyses of the blood of normal subjects, physicians, nurses, students and laboratory workers, of which only part have been published. 
and that of 28 undetermined acid fractions, 16.6 m.eq. Bicarbonate was 17.5, chloride 7.6 and base 9.2 m.eq. below, while undetermined acid was 13.0 m.eq. above, the normal average.

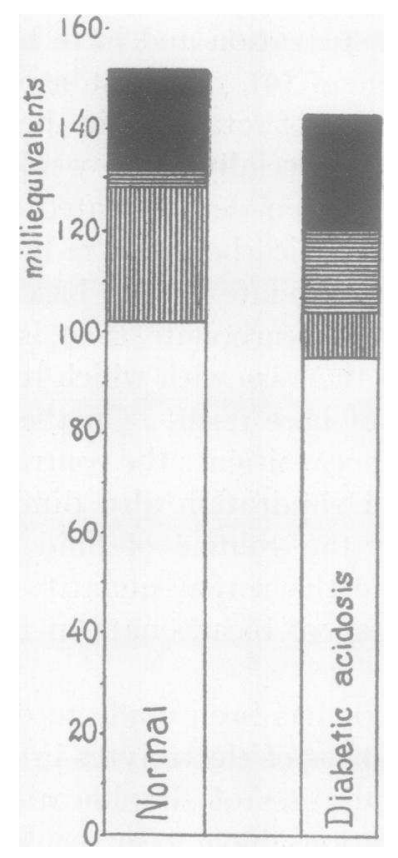

Fig 2. Diagrammatic Representation of the Chief Electrolyte and Water Changes Which Occur in the Body in Diabetic Acidosis

The vertical dimension in each column represents concentration in milliequivalents; the horizontal dimension, the relative volume of fluid in which the components are dissolved. In diabetic acidosis (right hand column) the volume of body fluid is depicted as 25 per cent below normal. The blank portions of the columns represent chloride; the vertically lined portions, bicarbonate; the horizontally lined portions, undetermined acid. Base is measured by the total height of each column.

\section{Description of development of acidosis}

The mechanism by which these electrolyte disturbances are probably produced has been described in some detail elsewhere. (1) $\mathrm{CO}_{2}$ is displaced from bicarbonate by ketone acids which accumulate in the body when carbohydrate is not burned. (2) A certain proportion of the ketone acids, because of their weak acid character, can be excreted as free acid in highly acid urine, and another fraction is neutralized by ammonia. None of these processes involves any irreversible reaction nor removes from the body any indispensable element. Up to this point combustion of the ketones alone will restore the normal constitution and reaction of the internal environment. The excretion of acid and am- 
monia may be considered as a defense against loss of body base. This defense is, however, not perfect. A certain fraction of the ketone acids in the urine is neutralized by fixed bases. The excretion of these bases, in contradistinction to responses 1 and 2, withdraws from the body elements which can be replaced only from extraneous sources and therefore introduces a reaction which is not automatically reversible. Given an adequate supply of proper bases in food to replace those lost in the urine, wastage might still be somewhat obviated. However, anorexia or even vomiting usually limit the intake of food. (4) Reduction of base means reduction of the total salt, or electrolyte, concentration of the body fluids. To meet this, fluid is excreted, with consequent dehydration. Apparently neither function, total fluid volume nor osmotic pressure, is sacrificed entirely in behalf of the other. Compromise between the two functions results in a lowered body fluid volume containing less than the normal concentration of base. (5) The reason for the loss of chloride is not clear and its utility seems questionable. In the urine $\mathrm{Cl}$ can be excreted only as the neutral salt of ammonia or some base. Its elimination would seem, therefore, inevitably to detract from the quantities of these bases which could otherwise serve the apparently more useful purpose of neutralizing the ketone acids. In vomitus chloride might be excreted as free hydrochloric acid without equivalent amounts of base. It would be easy to conclude, as has been suggested, that vomiting in acidosis was a protective mechanism, removing chloride to provide more base for the neutralization of ketone acids. The hypochloremia of diabetic acidosis is not related, however, to the severity of gastro-intestinal symptoms or the amount of vomiting and may be found in patients who have vomited little, if at all. Furthermore, in a large number of specimens of vomitus which have been analyzed no free hydrochloric acid has been found. In all, the concentration of base equalled or exceeded that of chloride. Loeb, Atchley, Richards and Benedict (17) found that severe diabetics from whom insulin had been withdrawn excreted in the urine large quantities of $\mathrm{Cl}$ even when ketosis did not develop, apparently from the diuretic effect of glycosuria alone. Chloride depletion, then, may be connected less with the acidosis than with the glycosuria and dehydration which accompany it. As might be expected, serum chloride and base deficits are usually associated, as if the chloride was lost chiefly as sodium chloride. This is well illustrated in the average figures which have been given above. The chloride deficit in this ideal diabetic acidosis was 7.6 m.eq., that of base 9.2 m.eq., indicating that the chloride had been lost entirely as $\mathrm{BCl}$, while in addition 1.6 m.eq. of base had been used to carry out ketone acids. Cases are encountered in which the chloride deficit far exceeds that of base, where $\mathrm{Cl}$ actually seems to have yielded some of its base for the neutralization of ketone acids. It has been inferred that in these cases chlorides as well as ketone acids have been excreted as ammonium salts. 
The 5 responses which give rise to the electrolyte pattern which is characteristic of diabetic acidosis have been discussed as if they occurred in a definite sequence, whereas they probably represent concurrent reactions. That the relative intensities of the different reactions follow no consistent rule is evident from the variety of patterns in Figure 3, which

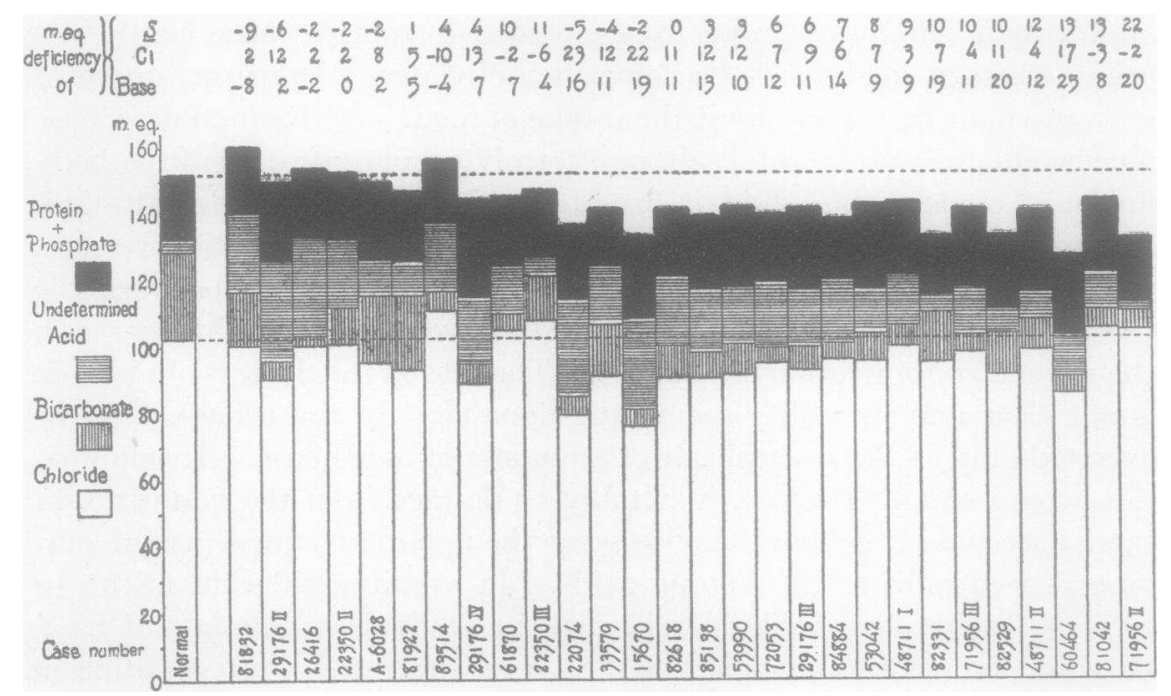

Fig. 3. Serum Electrolyte Patterns at the Height of Diabetic Acidosis

The concentrations of base and important acids at the time of the initial blood examination. The column on the extreme left gives, for comparison, the average normal serum electrolyte pattern. The broken lines traversing the chart represent the average normal concentrations of base and chloride. The figures at the top of the chart, representing deficiences of the three components of major interest, were derived by subtracting observed concentrations from average normal concentrations. Roman numerals after case numbers indicate different admissions of the same subject.

shows the initial serum electrolyte concentrations of patients who had complete acid base determinations. There is, nevertheless, a tendency for certain responses to be associated more frequently than others. With high undetermined acids, presumably ketones, for example, bicarbonate is usually low. In the 16 instances in which undetermined acids exceed 15 m.eq., bicarbonate was as low or lower than 10 m.eq. 12 times, and never exceeded 16 m.eq. This is to be expected in view of the fact that the primary cause of bicarbonate deficiency is accumulation of ketone acids. On the other hand, the converse is not equally true. In the 12 determinations in which undetermined acid was below 15 m.eq., bicarbonate exceeded $10 \mathrm{~m}$.eq. only 5 times. The reasons for the 7 exceptions are not hard to find. In 6 there was a base deficiency, twice coupled with 
normal or high chloride. In the remaining one base was at the lower and chloride at the upper limits of normal. There was, then, too little base uncombined with chloride to permit greater extension of the undetermined acid fraction.

There is a less close correlation between chloride and base reductions. With base above 145 m.eq., $\mathrm{Cl}$ exceeded 95 m.eq. 7 out of 9 times; but when base was below 145, $\mathrm{Cl}$ was above 95 in 9 of 19 observations. On the other hand, when $\mathrm{Cl}$ was below 95, base was below 14510 times out of 12 ; but when $\mathrm{Cl}$ was greater than 95 , base was less than 145 in 9 cases out of 16. This is confirmatory evidence that the $\mathrm{Cl}$ which is lost is excreted chiefly in combination with fixed base, while base is sacrificed partly in combination with ketone acids.

That the correlation between any two functions is extremely loose is evident from the great variety of patterns in the figure. In this figure an attempt has been made to arrange the studies in groups with more or less similar patterns; but, with four variables to consider, such a classification is quite unsatisfactory. Perhaps the most striking features are the infrequency with which chloride and base equal or exceed the average normal value. In only 2 instances (22350 III and 83514 ) is $\mathrm{Cl}$ actually above normal. The second of these can be dismissed with a word. The patient was admitted in coma following a cerebral hemorrhage, and glycosuria was only discovered later. Before the blood examination she had received, subcutaneously, $3000 \mathrm{cc}$. of salt solution. In the other studies, with high or normal chlorides, 22350 III, 61870, 81042 and 71956 II, entirely different situations presented themselves. Case 61870 is the only one in the series who gave no history of vomiting. Her history is, however, somewhat unreliable. Cases 22350 III and 81042 each vomited only once, but 71956 II had vomited repeatedly for 12 hours. The failure to deplete chloride can not, therefore, be ascribed entirely to absence of gastro-intestinal symptoms. In no one of the four was the high chloride attended by elevation of base. In all, then, there seems to have been predominantly a loss of base combined with bicarbonate or ketones, presumably the latter, and less than the usual sweeping out of chloride. The group included a case of maximum severity, $71956 \mathrm{II}$, as well as an early mild case, 22350 III. Judging from her nutritive state and history, 61870 had an essentially mild diabetes and would not have developed acidosis had it not been for a septicemia which arose from an infected finger. Case 81042 was a moderately severe case with slow, chronic development of acidosis.

In the first 10 studies of Figure 3 base was within or only slightly below the normal limits, at or above $145 \mathrm{~m} . e q$. In only one of the whole series, 81832 , was base actually above normal, $160.5 \mathrm{~m}$.eq. There are certain distinguishing characteristics about this group. In the first place it contains the only two studies in which $\mathrm{Cl}$ was above normal. There are, 
however, 4 with $\mathrm{Cl}$ below normal, A-6028, 81922, 29176 II and 29176 IV, one of whom, 81922 had not vomited. The series contains 4 of the mildest cases. However, 4 of the mildest are to be found to the right of the figure among those with low base. Moreover, among the first group with high base is 26416 , an extreme acidosis with a bicarbonate of only 3.2 m.eq. Perhaps the most consistent finding associated with high base is a high value for the sum of bicarbonate + undetermined acid, which will in the future be designated as $S$. In 8 of the 10 studies this value equalled or exceeded the normal 25 to $30 \mathrm{~m}$.eq. The remaining 2 are among the high chloride cases mentioned above. Undetermined acid, to the extent that it is composed of ketone acids, may be considered as base that may be made available for formation of bicarbonate through the instrumentality of insulin (or carbohydrate combustion) alone. If $S$ equals or exceeds the normal value, then, in theory bicarbonate concentration can be completely restored by the use of insulin alone. In some of the present studies, for example $81832, S$ is so high that combustion of ketones without other change would result in alkali excess. Undoubtedly in all cases body fluids have been lost so that the actual quantities of all acids and alkalies in the body are far less than the concentrations in the serum would suggest. The salt which has been lost with the fluid must have contained base and chloride in approximately the proportions in which they occur in normal serum. Although bicarbonate could be restored by insulin alone with, in many instances, enough extra bicarbonate to permit dilution, without reducing bicarbonate below normal limits, any degree of dilution would lower chloride, which is already at or below the normal limits. From the standpoint of the actual quantities of electrolyte in the body, then, the high base cases with high $S$ have suffered a disproportionately large chloride depletion.

Before passing on it may be well to call attention to the fact that changes in body fluid volume have a much greater effect on chloride and base than they do on bicarbonate concentration, because the total amounts of chloride and base in these fluids are larger. This can be easily illustrated by examples from the cases immediately under discussion. Case 81832 had 160.5 m.eq. of base, 100.5 of $\mathrm{Cl}$ and 38.9 m.eq. of $S$. If it be assumed that the 8.9 m.eq. of $S$ above the normal $30 \mathrm{~m} . e q$. resulted from concentration alone, the fluid volume must have been diminished by $100-\left(\frac{30.0}{38.9} \times 100\right)$, or 22.8 per cent. In this case the process could be reversed by a proportional reexpansion. This would restore $S$ to the normal level of 30.0 m.eq.; but would reduce $\mathrm{Cl}$ to the extremely low value of 77.6 m.eq. and base to only 123.8 m.eq. In other words, the production of a 9 m.eq. reduction of $S$ by dilution involves a reduction of 23 m.eq. of $\mathrm{Cl}$ and 37 m.eq. of $\mathrm{B}$. Compare with this the picture of 83514 . In this case $\mathrm{Cl}$ at $111.2 \mathrm{~m}$.eq. is about 9 m.eq. and base 
at 156.4 is 4 m.eq. above the normal average, while $S$ at 26.2 is just within the normal limits. To reduce $\mathrm{Cl}$ to normal the serum must be diluted $100-\left(\frac{102.0}{111.2} \times 100\right)=10$ per cent. This will bring base to 140.6 , $\mathrm{Cl}$ to 102.0 and $S$ to 23.6 m.eq. Dilution which lowers $\mathrm{Cl} 9$ m.eq., then, reduces base 16 m.eq., but $S$ only 2.6 m.eq.

The last 18 cases all present important base deficiencies, although they vary greatly in other respects. In only 2 instances, which have been discussed above, 81042 and 71956, is $\mathrm{Cl}$ above the normal average; in all others it is below. There is, however, little demonstrable parallelism between base and $\mathrm{Cl}$ levels in the whole group because of the associated variations of $S$. $S$ is normal or above in only 6 of the 18 observations. It goes without saying that these 4 have extremely low $\mathrm{Cl}$. It also follows that when $\mathrm{Cl}$ is relatively normal $S$ is greatly restricted. Again it is hard to connect the levels of base and $\mathrm{Cl}$ or the magnitude of $S$ with either vomiting or the severity of acidosis.

In the majority of instances distinctly low $S$ seems to be associated with more or less chronic or long standing acidosis. This is true in both the high base and low base series. Case 61870, with $S$ of 19.7 m.eq., and a diabetes of an essentially mild nature, had received no treatment till after she entered the hospital, two weeks after the onset of a finger infection, and 3 days after symptoms of acidosis had appeared and 24 hours after she had become stuporous. Case 81042 had neglected all treatment for a week before admission, developed a sore throat 4 days later, and had symptoms of acidosis 36 to 48 hours before entering the hospital. Case 71956 II had neglected treatment for 2 months and had symptoms of acidosis 48 hours before admission. The next time he entered the hospital (71956 III), he had neglected to take insulin for 3 days and symptoms of acidosis had persisted for 36 to 48 hours. On this occasion, also, diarrhea may have contributed to the reduction of base. Case 60464 , with a severe diabetes, had received no treatment of any value and had developed acidosis with no recognized complication. Case 82529 presents a rather peculiar situation. She also had neglected treatment until an intercurrent sore throat suddenly precipitated acidosis. This had been treated by injections of insulin alone, because stupor and vomiting prevented the oral administration of fluid. The insulin apparently partly overcame the ketosis, without otherwise benefiting the condition. These results suggest that long standing or chronic acidosis leads to gradual depletion of base combined with ketones, or available for combination with ketones. This is by no means an invariable rule. The remaining 2 cases with $S$ below 20 m.eq. (22350 III and 48711 II) had acidosis of extremely short duration and moderate severity which developed while they were under treatment. Moreover, in both, vomiting was a predominant symptom, although chloride was not decreased. Perhaps 
treatment enabled these subjects to excrete the ketone acids more rapidly in the urine.

It needs little argument to prove that with base low a large $S$ indicates extreme depletion of chloride. In this series there can be no reasonable doubt that chloride deficits far outweigh the reductions of base combined with or available for combination with bicarbonate. No amount of insulin, carbohydrate, or combination of the two could be expected under these conditions to restore a normal acid-base equilibrium. Again it must be emphasized that if there has been loss of fluid from the body-and presumably there has been in all these cases-the actual deficiency of chloride is far greater than the reduction of concentration would lead one to believe.

The relative deficiencies of base, chloride and $S$ have been determined by subtracting from the average normal values the concentrations of each of these components found in the serum at the height of acidosis. Values of these deficiencies can be found at the top of Figure 3. Both in frequency and extent base deficiencies are largest and $S$ deficiencies smallest. In the 28 cases the average deficiency of base is $9.3 \mathrm{~m}$.eq. and in only 3 instances is base above the normal average. The average deficiency of $\mathrm{Cl}$ is 6.9 m.eq. and only 3 observations are above the normal average. The average deficiency of $S$ is only 4.6, and 8 observations are above the normal average. Roughly, the effect of contraction of fluid volume on these components, being proportional to their concentrations, is, $\mathrm{B}: \mathrm{Cl}: S:: 150: 100: 30$. Therefore, any loss of body fluid which has taken place has diminished the apparent base deficit 5 times as much as it has the deficit of $S$. The effect of reexpansion of body fluid will affect base 5 times and $\mathrm{Cl} 3.33$ times as much as it will $S$. The reduction of $\mathrm{Cl}$ concentration covers two-thirds of the apparent base loss, but is equivalent to a smaller proportion of the actual base loss; the reduction of concentration of $\mathrm{Cl}$ is $\mathbf{5 0}$ per cent greater than that of $S$, but the actual deficiency of $\mathrm{Cl}$ must surpass that of $S$ by a much larger margin.

\section{DISCUSSION}

Although the serum electrolyte patterns in diabetic acidosis are infinitely diverse in detail, there is nothing in any of them incompatible with the theory of their production presented above. The diversity would seem to proceed from variation of the relative intensity of the 5 responses described, dependent probably on the prominence of certain associated symptoms (for example, vomiting), the severity of onset, the duration of the condition, the degree of body fluid loss, the functional efficiency of the kidneys and other less easily recognized factors. There are exceptions to every generalization which pretends to connect a given electrolyte pattern with any particular clinical aspect of the disease, presumably because the variables involved are too numerous to be managed. For 
the most part preservation of the base concentration of the serum seems to mark a relatively benign condition, but severe cases are encountered with little reduction of base, and mild cases with considerable base deficits. With high base, chloride is likely to be better preserved and the $S$ fraction is less often depleted. The electrolyte disturbance would seem to be more easily reversible.

Reduction of the $S$ fraction, which is equivalent to loss of base formerly combined with bicarbonate, is more commonly observed in long standing cases or cases who have a more or less chronic acidosis, although it has been observed in some of the mildest cases.

The outstanding facts are that in the great majority of cases of all kinds the concentration of chloride is reduced more than is the concentration of $S$. Accumulation of ketone acids at the expense of bicarbonate is undoubtedly the most striking feature of the serum electrolyte disorder. It is, however, reversible without the addition of any extraneous salt. Only when $S$ has been reduced by the excretion of base with ketones has the body been pauperized. It may be of some significance that, in the studies of Loeb, Atchley, Richards and Benedict (17) cited above, excretion of ammonia almost paralleled that of ketone acids. Chloride, however, was not covered by ammonia. In the most severe stages of acidosis encountered in clinical practice the defense against loss of fixed base with ketones would seem to be less perfect. Nevertheless, it still remains far superior to the mechanism for the neutralization of chloride.

Not only is the concentration of chloride reduced more than that of $S$, but the actual amounts of chloride lost must far exceed the concentration deficits if there is contraction of body fluids, for reasons that have been pointed out. Except for minor changes in phosphate and protein concentrations actual base losses must equal those of $\mathrm{Cl}$ and $S$ together and must exceed the losses of either individual fraction. That the estimated deficits given at the top of Figure 3 do not yield such equality can be attributed to the facts that deficits are calculated from arbitrary average figures, that fluctuations in the proteins and phosphates are not taken into consideration and that only concentrations of components are known. In most instances base deficits cover, or more than cover, those of $\mathrm{Cl}$, as one would expect if all chloride excreted was neutralized by fixed base. There are, however, certain exceptions to this rule, for example Cases 22074 and 15670 in Figure 3. In certain cases $S$ is little restricted and may even, as in these two extreme examples, exceed its normal magnitude. It would seem necessary to believe that in these cases enough ammonia had been excreted to neutralize not only the urinary ketones, but some chloride as well. Indeed this explanation has been offered in previous publications $(16,18,19)$ and mentioned above in explanation of these rather specific $\mathrm{Cl}$ reductions. It is, however, unnecessary to overtax ammonia production to such an incredible extent, if the effect of body 
fluid loss is again taken into consideration. Concentration of the total salts of the blood will, of course, raise base more than it will chloride. An example will illustrate the point. If, by loss of sodium chloride alone, the base of the serum were reduced from 150 to 140 and the chloride from 100 to 90 m.eq. and 10 per cent of the water in the serum were then removed, the base would increase to 154 , the chloride to only 99 . Determination of concentrations alone would now lead to the erroneous conclusion that the base had increased while chloride diminished. The apparent losses of $\mathrm{Cl}$ without coincident wastage of base may indicate only our present incapacity to include in our measurements three dimensions. Inferences drawn from studies of concentration only, even if these are accompanied by determinations of excretion of the components involved, must give incomplete information. Some attempt must be made to estimate, in addition, the volume of fluid in which each component is distributed within the body.

\section{CONCLUSIONS}

The nature and mode of production of the abnormal serum electrolyte patterns in diabetic acidosis have been discussed from a theoretical standpoint. The patterns actually observed in a considerable number of cases have been analyzed and found compatible with the processes suggested. These patterns are characterized by extreme diversity which can not always be clearly related to distinctive features of the disease. It has been pointed out that base from which bicarbonate has been displaced by ketones within the body may be considered as base available for combination with bicarbonate by simple reversal of the disorder of carbohydrate metabolism which led to ketosis. Base sacrificed in the urine with ketones can only be restored from extraneous sources. The amount of base lost in this manner, however, in the great majority of cases falls far short of the quantity sacrificed with chloride. The actual deficiency of body chloride is far greater than concentration deficits would indicate if there is associated loss of body fluid, and far exceeds the deficiency of bicarbonate, if in the latter is included the potential bicarbonate represented by the fraction of base combined with ketones.

\section{BIBLIOGRAPHY}

1. Walter, F., Arch. f. exper. Path. u. Pharmakol., 1877, vii, 148 . Untersuchungen über die Wirkung der Säuren auf den thierischen Organismus.

2. Fields, H., Jr., Personal communication cited by Peters and Van Slyke, Quantitative Clinical Chemistry. I. Interpretations. Williams and Wilkins Co., Baltimore, 1931, p. 170.

3. Hartmann, A. F., and Darrow, D. C., J. Clin. Invest., 1928, vi, 257. Chemical Changes Occurring in the Body as the Result of Certain Diseases. III. The Composition of the Plasma in Severe Diabetic Acidosis and the Changes Taking Place during Recovery.

4. Deuel, H. J., Jr., and Gulick, M., J. Biol. Chem., 1930, lxxxix, 93. The Relation between Alkali Deficit and Glucose Tolerance in the Dog. 
5. Haldane, J. B. S., Lancet, 1924, i, 537. Experimental and Therapeutic Alterations of Human Tissue Alkalinity.

6. Beumer, H., and Soecknick, A., Ztschr. f. Kinderh., 1924, xxxvii, 236. Über organische Acidose bei anorganischer Acidose und Alkalose.

7. Porges, O., and Lipschütz, H., Arch. f. exp. Path. u. Pharmakol., 1923, xcvii, 379. Über Azetonurie und Alkalose.

8. Adlersberg, D., Biochem. Ztschr., 1923, cxliii, 527. Acetonurie und Acidose.

9. Davies, H. W., Haldane, J. B. S., and Kennaway, E. L., J. Physiol., 1920, liv, 32. Experiments on the Regulation of the Blood's Alkalinity.

10. Booher, L. E., and Killian, J. A., Proc. Soc. Exper. Biol. and Med., 1924, xxi, 528. Ketosis Associated with Conditions of Alkalosis.

11. Grant, S. B., and Goldman, A., Am. J. Physiol., 1920, lii, 209. A Study of Forced Respiration: Experimental Production of Tetany.

12. Peters, J. P., Wakeman, A. M., Eisenman, A. J., and Lee, C., J. Clin. Invest., 1929, vi, 517. Total Acid-base Equilibrium of Plasma in Health and Disease. X. The Acidosis of Nephritis.

13. Bulger, H. A., Peters, J. P., Lee, C., and Murphy, C. F., Arch. Int. Med., 1925, xxxvi, 857. The Concentration of the Blood and of the Urine in Diabetic Toxemia.

14. Peters, J. P., Kydd, D. M., and Eisenman, A. J., J. Clin. Invest., 1933, xii, 355. Serum Proteins in Diabetic Acidosis.

15. Gamble, J. L., Ross, G. S., and Tisdall, F. F., J. Biol. Chem., 1923, Ivii, 633. The Metabolism of Fixed Base during Fasting.

16. Peters, J. P., Yale J. Biol. and Med., 1930, ii, 183. Acid-base Equilibrium and Salt and Water Exchange.

17. Loeb, R. F., Atchley, D. W., Richards, D. W., Jr., and Benedict, E. M., J. Clin. Invest. (Proc.), 1931, x, 664. A Study of the Electrolyte Metabolism in Diabetic Acidosis Induced in Human Subjects.

18. Peters, J. P., Bulger, H. A., Eisenman, A. J., and Lee, C., J. Clin. Invest., 1926, ii, 167. Total Acid-base Equilibrium of Plasma in Health and Disease. VI. Studies of Diabetes.

19. Peters, J. P., and Van Slyke, D. D., Quantitative Clinical Chemistry. I. Interpretations. Williams and Wilkins, Baltimore. 1931.

20. Allen, F. M., Stillman, E., and Fitz, R., Monographs of the Rockefeller Institute for Medical Research, No. 11, October 15, 1919 (see especially page 450). Total Dietary Regulation in the Treatment of Diabetes. 